\title{
Biomimetic Artificial Epigenetic Code for Targeted Acetylation of Histones
}

\section{$\operatorname{AUTHOR(S):~}$}

Taniguchi, Junichi; Feng, Yihong; Namasivayan, Ganesh Pandian; Hashiya, Fumitaka; Hidaka, Takuya; Hashiya, Kaori; Park, Soyoung; Bando, Toshikazu; Ito, Shinji; Sugiyama, Hiroshi

\section{CITATION:}

Taniguchi, Junichi ...[et al]. Biomimetic Artificial Epigenetic Code for Targeted Acetylation of Histones. Journal of the American Chemical Society 2018, 140(23): 7108-7115

\section{ISSUE DATE:}

2018-05-24

URL:

http://hdl.handle.net/2433/243836

\section{RIGHT:}

This document is the unedited Author's version of a Submitted Work that was subsequently accepted for publication in Journal of the American Chemical Society, copyright (c) American Chemical Society after peer review. To access the final edited and published work see https://doi.org/10.1021/jacs.8b01518.; This is not the published version. Please cite only the published version.; この論文は出版社版でありません。引用の際には出版社版をご確認ご利用ください。 


\title{
Biomimetic artificial epigenetic code for targeted acetylation of histones
}

\author{
Junichi Taniguchi, ${ }^{\dagger}$ Yihong Feng, ${ }^{\dagger, \#}$ Ganesh N. Pandian, ${ }^{\ddagger,}{ }^{*}$ Fumitaka Hashiya, ${ }^{\dagger}$ Takuya Hidaka, ${ }^{\dagger}$ \\ Kaori Hashiya, ${ }^{\dagger}$ Soyoung Park, ${ }^{\dagger}$ Toshikazu Bando, ${ }^{\dagger}$ Shinji Ito, ${ }^{\S}$ and Hiroshi Sugiyama*,+,‡ \\ ${ }^{\dagger}$ Department of Chemistry, Graduate School of Science Kyoto University, Sakyo-Ku, Kyoto 606-8502, Japan \\ ${ }^{\ddagger}$ Institute for Integrated Cell-Material Sciences, Institute for Advanced Study, Kyoto University, Sakyo-Ku, Kyoto \\ 6o6-8502, Japan \\ \$Medical Research Support Center, Graduate School of Medicine, Kyoto University, Sakyo-Ku, Kyoto 6o6-8501, Ja- \\ pan \\ \#These authors contributed equally to this study.
}

\begin{abstract}
While the central role of locus-specific acetylation of histone proteins in eukaryotic gene expression is well established, the availability of designer tools to regulate acetylation at particular nucleosome sites remains limited. Here, we develop a unique strategy to introduce acetylation by constructing a bifunctional molecule designated Bi-PIP. Bi-PIP

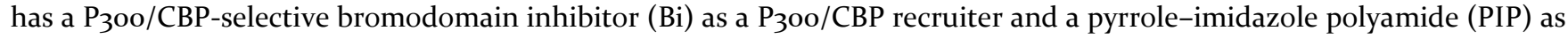
a sequence-selective DNA binder. Biochemical assays verified that Bi-PIPs recruit $\mathrm{P}_{300}$ to the nucleosomes having their target DNA sequences and extensively accelerate acetylation. Bi-PIPs also activated transcription of genes that have corresponding cognate DNA sequences inside living cells. Our results demonstrate that Bi-PIPs could act as a synthetic programmable histone code of acetylation, which emulates the bromodomain-mediated natural propagation system of histone acetylation to activate gene expression in a sequence-selective manner.
\end{abstract}

\section{INTRODUCTION}

Posttranslational modifications (PTMs) on histone proteins play significant roles in epigenetic regulation of eukaryotic chromatin. Acetylation of epsilon-amine on lysine residues is one of the major PTMs on histones, and is strongly correlated with transcriptional activation. The code governing histone acetylation is regulated by histone acetyltransferases (HATs) as writers and by histone deacetylases (HDACs) as erasers. Acetylated histones are known to operate via two mechanisms. One is to decrease the positive charge of histone proteins, resulting in weakened interaction between histones and DNA, which in turn open up the chromatin structure. ${ }^{1}$ The second is mediated by bromodomain (BD)-containing proteins, which selectively bind to acetylated lysine residues. ${ }^{2,3}$

Although locus-specific (i.e., DNA sequence-specific) regulation of histone acetylation has been shown to be essential in biological processes, techniques for controlling histone acetylation at any region of interest remain limited. Recently, programmable DNA-binding module proteins such as transcription activator-like effectors (TALEs) and clustered regularly interspaced short palindromic repeat (CRISPR)/Cas systems were fused to the catalytic domain of histone acetyltransferase $\mathrm{P}_{3} 00$ for sequence-selective histone acetylation. ${ }^{4}$ Although targeted acetylation was demonstrated using the dCas9- $\mathrm{P}_{3}$ oo Core fusion protein, the transfection-based system could limit their clinical applications. Moreover, such protein-based molecules are amenable to enzymatic degradation in the cellular environment. In these contexts, the development of chemically synthesized sequence-selective molecules is a promising alternative for locus-specific acetylation of histones, avoiding the need of transfection and enzymatic degradation.

Pyrrole-imidazole polyamides (PIPs) are a class of synthetic molecules that bind to DNA in a sequence-selective manner. ${ }^{5}$ In the anti-parallel hairpin structure, a pair of pyrrole and pyrrole $(\mathrm{Py} / \mathrm{Py})$ recognizes $\mathrm{A} \cdot \mathrm{T}$ or $\mathrm{T} \cdot \mathrm{A}$ base pair, while a pair of imidazole and pyrrole $(\mathrm{Im} / \mathrm{Py})$ does $\mathrm{G}$ - $C$ base pair. ${ }^{6}$ It is known that the aliphatic $\beta$-alanine can substitute and partake the selective binding functionality of the Py in the PIP chemical architecture. ${ }^{7}$ Also, $\beta$-alanine could confer the PIPs with better flexibility and improved binding affinity. By conjugating PIP with a HDAC inhibitor SAHA, our group previously developed a class of designer molecule called SAHA-PIP for sequence-selective histone acetylation. ${ }^{8}$ SAHA-PIPs with different sequence-specificity activated silenced genes including pluripotency genes, ${ }^{9,10}$ germ cell-associated genes, ${ }^{11}$ retinal genes, ${ }^{12}$ neural genes, ${ }^{13}$ and key therapeutically important genes ${ }^{14}$ in human dermal fibroblasts. Despite the proclaimed potential of SAHA-PIPs as epigenetic drugs, the level of gene activation was still inconsistent. One reason for this observation could be the fact that induction of histone acetylation by inhibiting HDACs is an indirect outcome and acetylation will not occur if HATs are absent in the region. As an alternative approach, a $\mathrm{P}_{3} \mathrm{Oo}$ activator $\mathrm{N}$-(4-chloro-3-trifluoro- 


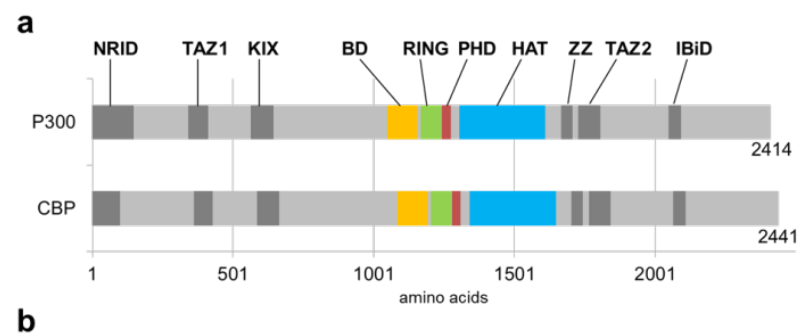

b
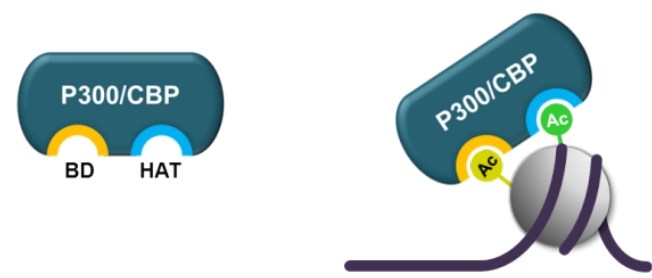

C
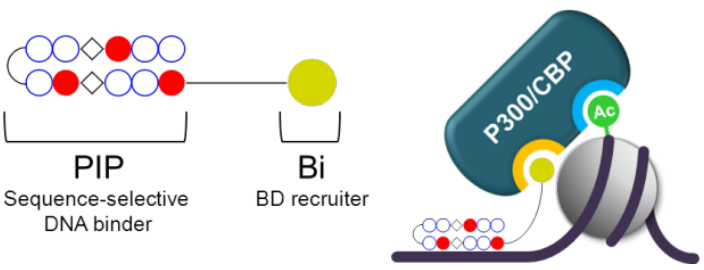

Figure 1. Strategy of targeted histone acetylation utilizing the bromodomain (BD)-mediated propagation of acetylation. (a) Structure of $\mathrm{P}_{300 / C B P}$ coactivator proteins. NRID, nuclear receptor interacting domain; TAZ1, transcriptional adaptor zinc finger 1; KIX, KID-interacting; BD, bromodomain; RING, really interesting new gene; PHD, plant homeodomain; HAT, histone acetyltransferase; ZZ, ZZ-type zinc finger; TAZ2, transcriptional adaptor zinc finger 2; IBiD, interferon-binding domain. (b) BD-mediated propagation of histone acetylation by $\mathrm{P}_{300 / C B P}$. Existing acetyl lysine (yellow) is recognized by BD of $\mathrm{P}_{300 / C B P}$, and de novo acetyl modification (green) is introduced by HAT domain. (c) Design of artificial epigenetic code of acetylation named 'Bi-PIP' (left) and model of targeted histone acetylation by Bi-PIP (right). Bi mimics acetyl lysine to recruit $\mathrm{P}_{300 / C B P}$ through its $\mathrm{BD}$.

methyl-phenyl)-2-ethoxy-benzamide (CTB ${ }^{15}$ was conjugated to PIPs. ${ }^{16}$ However, the mechanism by which CTB operates is yet to be clarified and there is no clear evidence to substantiate the interaction of CTB with P30o. ${ }^{17}$ Therefore, there is a need for (1) a more reliable and robust molecular design, and (2) proof-of-concept experiments of the molecular mechanism, to achieve sequence-selective histone acetylation in a similar manner to that observed in natural cellular environment.

Interestingly, HAT domain and BD sometimes appear in the same protein. In humans, $\mathrm{GCN}_{5}$ (KAT2A), PCAF (KAT2B), $\mathrm{P}_{300}$ (KAT3 $\left.\mathrm{B}\right), \mathrm{CBP}\left(\mathrm{KAT}_{3} \mathrm{~A}\right), \mathrm{TAF}_{1}\left(\mathrm{KAT}_{4}\right)$, and $\mathrm{BRD}_{4}$ contain both HAT and $\mathrm{BD}$ in their structures (Figure 1a). ${ }^{18-21}$ This is thought to contribute to the robust maintenance of highly acetylated chromatin; one acetyl mark causes acetylation of neighboring sites via BDmediated recruitment of these proteins (Figure 1b). ${ }^{18,22,23}$ This natural system inspired us to develop a novel approach based on the BD-mediated acetylation to acetylate histones in targeted nucleosome.
Small molecules targeting the $\mathrm{BD}$-containing proteins have been identified and developed in recent years. ${ }^{24-28}$ These BD inhibitors specifically bind to the pockets of BD to prevent binding with an acetylated lysine residue. Therefore, BD inhibitors could be used as a mimetic of acetylated lysine to probe BDs.

We hypothesized that locus-specific acetylation by a HAT-BD protein such as $\mathrm{P}_{300}$ could be achieved when a $\mathrm{BD}$ inhibitor is localized to a specific DNA sequence (Figure 1c). Herein, we report the development of a novel molecule named 'Bi-PIP' by conjugating a BD inhibitor (Bi) and PIP. We demonstrate a proof-of-concept that Bi-PIP can enhance histone acetylation by $\mathrm{P}_{3} 00$ in a sequence-selective manner and clarify their mode of action.

\section{RESULTS}

Design and synthesis of Bi-PIP as a sequence-specific artificial histone code of acetylation. We selected the coactivator $\mathrm{P}_{300 / C B P}$ family of proteins as the target for the artificial histone code (Figure 1a). $\mathrm{P}_{300 / C B P}$ plays a central role in transcriptional activation in eukaryotic cells. ${ }^{29}$ Given that $\mathrm{P}_{300 / C B P}$ has both HAT and BD, BDmediated propagation of histone acetylation was proposed (Figure 1b). ${ }^{18} \mathrm{~A} \mathrm{P}_{300 / C B P}$-selective $\mathrm{BD}$ inhibitor ( $\left.\mathrm{CBP}_{30}\right)$ having 5-isoxazolyl-benzimidazole was previously developed by Hay et al. (Supplementary Figure 1). ${ }^{25} \mathrm{X}$-ray crystal structure analyses revealed that the 3,5-dimethylisoxazole moiety in $\mathrm{CBP}_{3}$ o enters the pocket in the $\mathrm{BD}$ of $\mathrm{CBP}$ and $\mathrm{P}_{300}$, mimicking acetylamide of epsilon-acetylated lysine residue. ${ }^{25,30}$ The other two domains, terminal aryl group and morpholine moiety, are located outside of the pocket, suggesting that they could be sites for conjugation with PIPs (Supplementary Figure 1). $3^{\circ}$ For synthetic accessibility of building blocks, we chose one of the $\mathrm{CBP}_{30}$ derivatives (1 in Figure 2, named 'Bi' in this study), which showed a moderate binding affinity to the BD of CBP (Figure 2, Supplementary Figure 2) and attempted to attach a linker domain on the methoxy group. ${ }^{25} \mathrm{~A}$ BD inhibitor unit (Bi Unit) with primary amine was then designed and synthesized (Supplementary Scheme 1). By coupling with PIPs, which has a carboxylic acid at the N-terminus, two conjugates, termed Bi-PIP1 (2) and Bi-PIP2 (4) targeting $5^{\prime}-\mathrm{WWCWGWCW}-3^{\prime}$ and $5^{\prime}-\mathrm{WGCCGCCW}-3^{\prime}$, respectively, were synthesized ( $\mathrm{W}=\mathrm{A}$ or $\mathrm{T}$, Figure $\mathbf{2})$.

Bi-PIPs promote histone acetylation on nucleosomes that possess their target DNA sequences. To test sequence-selective histone acetylation by Bi-PIP, we sought to establish a biochemical assay by combining HAT reaction followed by immunoprecipitation using reconstituted nucleosomes reported previously ${ }^{22}$ and quantitative polymerase chain reaction (qPCR). We reconstituted mononucleosomes using a salt-dialysis method ${ }^{31}$ from a histone octamer and DNA templates that each have a Widom 601 nucleosome positioning sequence ${ }^{32}$ and five PIP-binding sequences: 5'-AACAGTCA-3' for $\mathrm{Bi}^{\prime}$ PIP1 and 5'AGCCGCCA-3' for Bi-PIP2 (Figure 3a). Gel electrophore- 

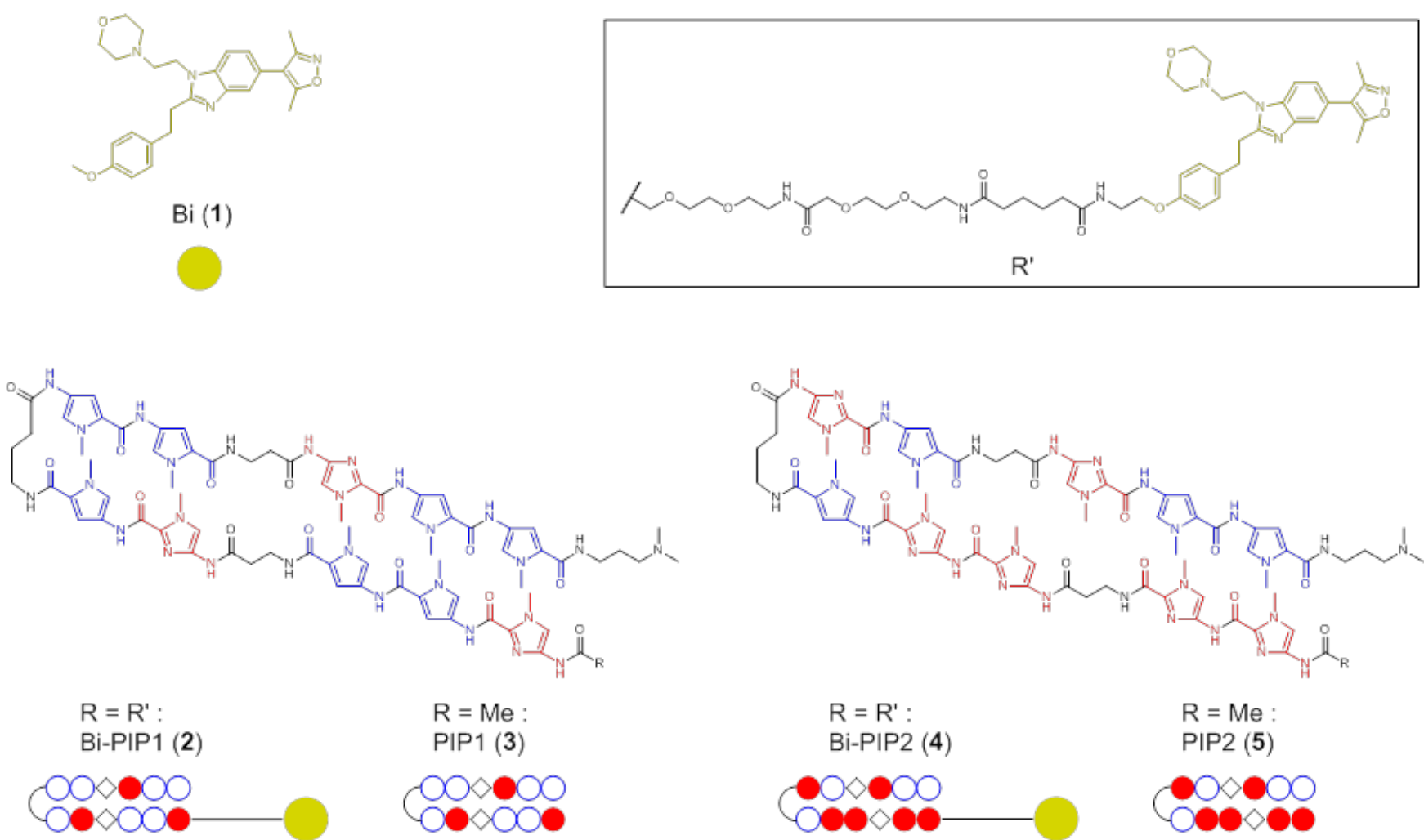

Figure 2. Chemical structures of Bi (1), Bi-PIP1 conjugate (2), PIP1 monomer (3), Bi-PIP2 conjugate (4), and PIP2 monomer (5).

sis confirmed that the majority of DNA templates were successfully formed on the nucleosome (Supplementary Figure 3). Two types of nucleosomes, one with five match sequences for Bi-PIP1 (Nuc1) and the second for Bi-PIP2 (Nuc2), were mixed and incubated with recombinant human $\mathrm{P}_{300}$ (amino acid 965-1810) including the BD and HAT with or without Bi-PIP1. The reaction mixture was then immunoprecipitated using an antibody for pan-acetylated $\mathrm{H}_{3}$ (acetylation at $\mathrm{K}_{9}, \mathrm{~K}_{14}, \mathrm{~K}_{1} 8, \mathrm{~K}_{23}, \mathrm{~K}_{27}$ ), followed by qPCR analysis to measure the amount of each nucleosome (Figure $\mathbf{3 b}$ ).

In the absence of the Bi-PIP, the level of acetylation of the nucleosomes got slightly increased only in the presence of $100 \mathrm{nM}$ of $\mathrm{P}_{300}$. On the other hand, addition of $100 \mathrm{nM}$ $\mathrm{Bi}-\mathrm{PIP} 1$ induced intensive acetylation on its target Nucr but not on Nuc2 (Figure 3c). The increase in the level of acetylation was dependent on the concentration of $\mathrm{P}_{300}$. Next, to clarify the importance of the Bi and PIP domains, each monomer molecule was tested in the assay. The PIP monomer alone (3), Bi monomer alone (1), and even a mixture of them could not promote the acetylation (Figure $3 \mathbf{d}$ ). The result clearly indicates that the Nuc1-selective acetylation by $\mathrm{Bi}-\mathrm{PIP} 1$ was achieved by sequence-selective binding of the PIP domain to its target DNA sequence and $\mathrm{P}_{300}$ recruitment mediated by a BD-Bi interaction (Figure 1c). To examine the programmability of Bi-PIP, we tested Bi-PIP2 designed to target Nuc2. Bi-PIP2 caused selective acetylation of Nuc2, indicating that the target sequence of Bi-PIP is programmable (Figure $\mathbf{3 e}$ ).
Taken together, we established that HAT reaction followed by in vitro ChIP-qPCR could be used to quantify the acetylation of individual nucleosomes, thereby demonstrating a proof-of-concept of sequence-selective acetylation with Bi-PIP using the assay.

Next, to investigate the effect of linker length of Bi-PIP, Bi-PIP1 with a shorter linker (Bi-PIP1-short, 6) and one with a longer linker (Bi-PIP1-long, 7) were synthesized (Supplementary Figure 4a) and compared with the original Bi-PIP1 in the assay. Acetylation of Nuc1 was enhanced at a similar level in Bi-PIP1-short, Bi-PIP1, and Bi-PIP-long (Supplementary Figure $\mathbf{4 b}$ ). The result suggests that all three Bi-PIPs have a sufficiently long and flexible linker for the effective acetylation of $\mathrm{H}_{3}$. Indeed, the $\mathrm{N}$-terminal tail region of $\mathrm{H}_{3}$ is known to be located near the linker DNA,33 where the binding sites for PIPs are located.

To clarify the binding site(s) essential for the acetylation, we carried out studies using five additional templates that have a single binding site for Bi-PIP1 at different positions (Nuc3-7). Among the five nucleosomes, Nuc3, which has a binding site at the closest position to the nucleosome core particle, showed the highest level of acetylation (Supplementary Figure 5). Thus, this is the most effective position for the acetylation. However, to our surprise, the acetylation level of Nuc3 was much lower than that of Nuc1 (Supplementary Figure 5). Moreover, even the sum of acetylation levels of Nuc3-7 did not reach the value for Nuc1, suggesting the existence of synergistic effects of multiple $\mathrm{P}_{3}$ oos on a single nucleosome (Supplementary Figure 5). Although this phenomenon could be explained by 
a
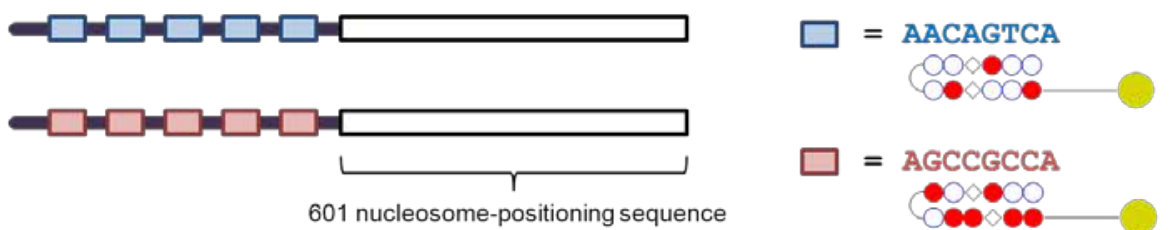

b
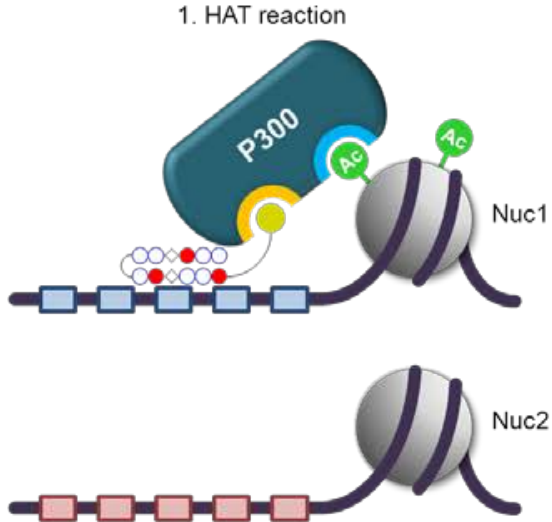

C

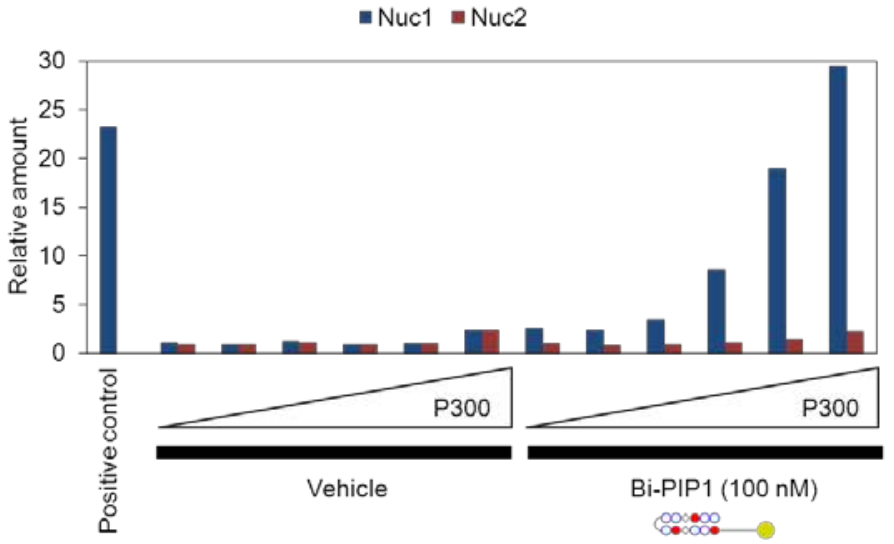

d

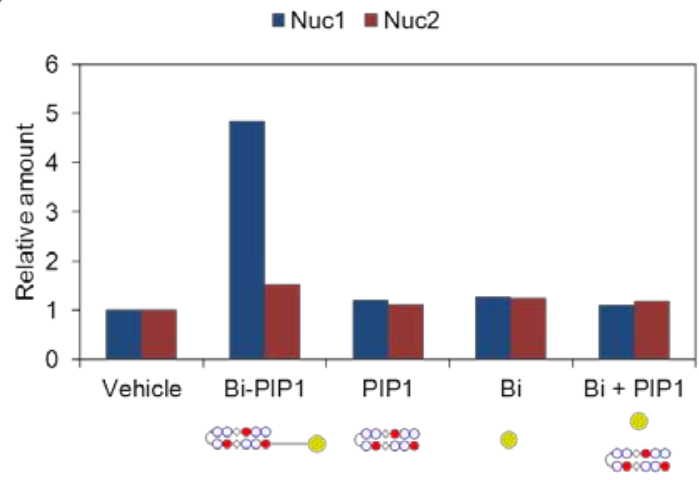

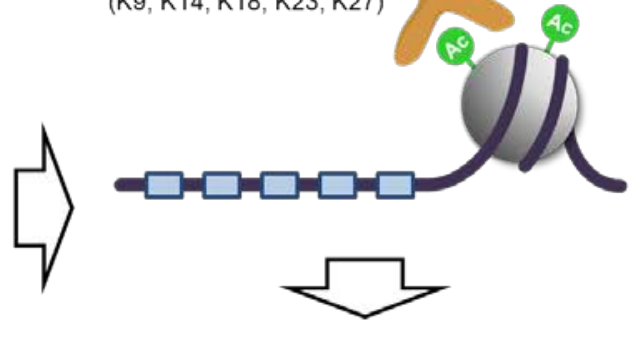

3. $\mathrm{qPCR}$

2. Immunoprecipitation

Anti-acetylH3

(K9, K14, K18, K23, K27)

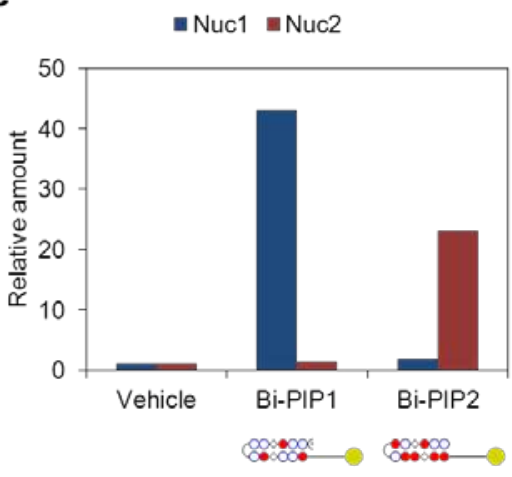

Figure 3. Bi-PIPs promote histone acetylation on nucleosomes that possess their target DNA sequences. (a) Schematic of DNA templates used for the assay. Blue and red rectangles represent binding sites for Bi-PIP1 and Bi-PIP2, respectively. (b) Schematic of HAT reaction-in vitro ChIP-qPCR. (c) A HAT reaction-in vitro ChIP-qPCR was performed with a series of $\mathrm{P}_{300}$ concentration $(\mathrm{o}, 3,10,30,100 \mathrm{nM})$ with or without $100 \mathrm{nM}$ of Bi-PIP1. Positive control represents a nucleosome with synthetic acetylated histone $\mathrm{H}_{3}$ (K4ac, K9ac, K14ac, K18ac, K23ac). (d) A HAT reaction-in vitro ChIP-qPCR was performed with 10 nM of P3oo. Each compound was applied at a concentration of $100 \mathrm{nM}$. (e) A HAT reaction-in vitro ChIP-qPCR was performed Bi-PIP1 (100 nM) or Bi-PIP2 (100 $\mathrm{nM}$ ) with $10 \mathrm{nM}$ of $\mathrm{P}_{300}$ 
the previously reported intermolecular auto-acetylation of $\mathrm{P}_{300,34,35}$ further studies is required to investigate the mechanism in detail.

Finally, histone acetylation with Bi-PIP was directly confirmed by mass spectrometry analysis. After HAT reaction, the histones were processed through propionylation of unmodified lysine, tryptic digestion, and propionylation of terminal amine. ${ }^{36,37}$ The resulting peptides were analyzed by liquid chromatography-tandem mass spectrometry (LC-MS/MS). Consistent with the antibody-based assays shown above, an increase in the signal intensity was observed for peptides containing acetyl lysine at the $\mathrm{N}$-terminal tail region of $\mathrm{H}_{3}$, such as $\mathrm{H}_{3} \mathrm{~K}_{1} 4 \mathrm{ac}, \mathrm{H}_{3} \mathrm{~K}_{1} 8 \mathrm{ac}, \mathrm{H}_{3} \mathrm{~K}_{2} 3 \mathrm{ac}$, $\mathrm{H}_{3} \mathrm{~K}_{2} 7 \mathrm{ac}$, and $\mathrm{H}_{3} \mathrm{~K}_{3} 6 \mathrm{ac}$ (Supplementary Figure 6a, 6c). It was also found that acetylation was promoted in the $\mathrm{N}$ terminal region of histone $\mathrm{H}_{4}$, including $\mathrm{H}_{4} \mathrm{~K}_{5} \mathrm{ac}, \mathrm{H}_{4} \mathrm{~K} 8 \mathrm{ac}$, and $\mathrm{H}_{4} \mathrm{~K}_{2}$ ac (Supplementary Figure 6b, 6d). The acetylation at $\mathrm{N}$-terminal tail region of $\mathrm{H}_{3}$ and $\mathrm{H}_{4}$ by $\mathrm{P}_{3} \mathrm{Oo}$ is consistent with previously reported results, where $\mathrm{P}_{300}$ gets recruited to reconstituted chromatin by DNA-binding activator proteins. ${ }^{8}$

Bi-PIPs epigenetically activate selective gene expression inside living cells. We then evaluated if Bi-PIPs could function in a cellular environment. As an initial experiment, we performed a HAT reaction-in vitro ChIPqPCR assay using HeLa nuclear extract as the HAT resource instead of recombinant $\mathrm{P}_{300}$. The nuclear extract containing not only $\mathrm{P}_{300 / C B P}$ but also other HATs and HDACs, provided a cell-like environment than that observed with only recombinant $\mathrm{P}_{300}$. Although the basal level of acetylation without Bi-PIPı increased, Bi-PIP1 enhanced acetylation of Nuc1 but not Nuc2 (Supplementary Figure 7), suggesting the possibility of applying Bi-PIPs to living cells.

A bottleneck in the utility of Bi-PIPs is the potential of $\mathrm{Bi}$ to impair the natural function of $\mathrm{P}_{300 / C B P}$ such as the propagation of histone acetylation inside the living cells. In this regard, we evaluated the effect of increasing amount of $\mathrm{Bi}$ on the BD-mediated histone acetylation. Interestingly, even a ten-fold higher concentration of Bi than that of BiPIP1 did not affect the BD-mediated acetylation induced by Bi-PIP1 (Supplementary Figure 8). It is reasonable to assume that the binding of $\mathrm{BD}$ and $\mathrm{Bi}$ could be in equilibrium, which provides the BD with the opportunity to interact with Bi-PIP1 even with the high doses of $\mathrm{Bi}$. The rapid dissociation of $\mathrm{CBP}_{3}$ from $\mathrm{P}_{3}$ oo further supports this notion. $3^{30}$

We then investigated the cellular activity of Bi-PIPs using the HEK293T cell line. The cell permeability of PIPs is known to vary depending on the chemical structures and cell lines.39,40 To ensure that all the compounds enter the cells, we used a delivery reagent, Endo-Porter. ${ }^{4} \mathrm{We}$ found that the inclusion of Endo-Porter greatly improved cellular uptake of a fluorescent PIP whose permeability is poor without the reagent (Supplementary Figure 9).

Considering the fundamental role of $\mathrm{P}_{300 / C B P}$ as the transcriptional coactivator to activate gene expression, we performed a transcriptome analysis of total RNA extracted from cells treated with the compounds. The criteria of $>2$ or $<-2$ fold change and $<0.05 \mathrm{P}$ value was applied for detecting differentially expressed transcripts. The Bi monomer showed only a minimal change in gene expression (2 upregulated transcripts and 4 downregulated transcripts). The PIP1 and PIP2 monomers caused a moderate change in global gene expression (32 up- and 31 downregulated by PIP1, and 143 up- and 63 downregulated by PIP2). On the other hand, Bi-PIP1 and Bi-PIP2 conjugates gave greater transcriptome changes, mainly for activation, rather than repression ( 473 up- and 258 downregulated by Bi-PIP1, and 446 up- and 137 downregulated by Bi-PIP2, Supplementary Figure 10). The results, therefore, reflect the recruitment of $\mathrm{P}_{300 / C B P}$ onto the chromatin by Bi-PIPs, which cannot occur by Bi monomer or PIP monomers.

We then evaluated if the difference in DNA sequence-selectivity could cause a dissimilar change in gene expression. In contrast to the in vitro ChIP assay shown above, genes activated by the two Bi-PIPs were not exclusive, and 247 transcripts were commonly upregulated by both Bi-PIP1 and Bi-PIP2 (Supplementary Figure 11). This is reasonable because the binding sites of Bi-PIP1 and Bi-PIP2 are not exclusively distributed throughout the genome; thus, some genes could be activated by both Bi-PIP1 and Bi-PIP2. To check whether the sequence-selective activity attributes to the the cellular function of Bi-PIPs, ChIP-sequencing (ChIP-seq) was performed using the antibody for pan-acetylated $\mathrm{H}_{3}$. Mapped reads were processed by One-stage DIffereNtial peak caller (ODIN) ${ }^{42}$ to detect differential peaks between Bi-PIP1 and Bi-PIP2-treated cells. Analysis of the target sequences in the region of differential peaks revealed that the target sequence of Bi-PIPı appeared more frequently in the loci where Bi-PIP1 showed higher peak signal than that in Bi-PIP2 (Figure 4a, left). Conversely, the target sequence of $\mathrm{Bi}-\mathrm{PIP}_{2}$ got enriched in the loci where Bi-PIP2 showed higher peak signal than that in BiPIP1 (Figure 4a, right). The enrichment of the target sequence for Bi-PIP1 was relatively lower than that for $\mathrm{Bi}-$ $\mathrm{PIP}_{2}$, which could be attributed to the more extensive sequence match sites for the Bi-PIP1 than Bi-PIP2 (8 fold in theory because it has three more $\mathrm{W}$ recognition). Therefore, it is reasonable to assume that the Bi-PIP1 could distribute more broadly and the binding sequence might be often found in the differential peak region for Bi-PIP2. Taken together, the observed epigenome changes in acetylation verifies the sequence-selective bioactivity of $\mathrm{Bi}$ PIPs.

Although the result above showed that some genes were activated by both Bi-PIP1 and Bi-PIP2, differentially activated transcripts were also identified in the microarray data by applying criteria of $>1.5$ or $<-1.5$ fold change (Figure $4 \mathbf{b}$, Supplementary Table 4, 5). Among these uniquely activated transcripts, the most upregulated protein-coding genes with Bi-PIP1 and Bi-PIP2 were NTS (neurotensin) and IER5L (immediate early response 5-like), respectively (Figure $\mathbf{4} \mathbf{b}$, Supplementary Table 4, 5). We verified the expression of these two transcripts using RTqPCR (Figure 4c). To gain insights into the mechanism of activation, we searched for the target sequences of Bi-PIP1 
a

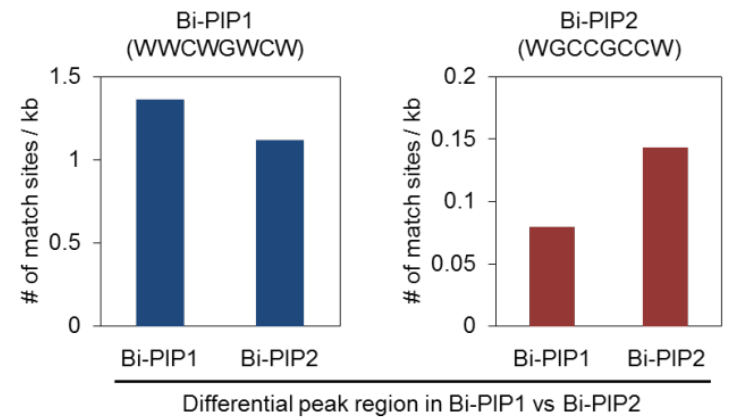

b

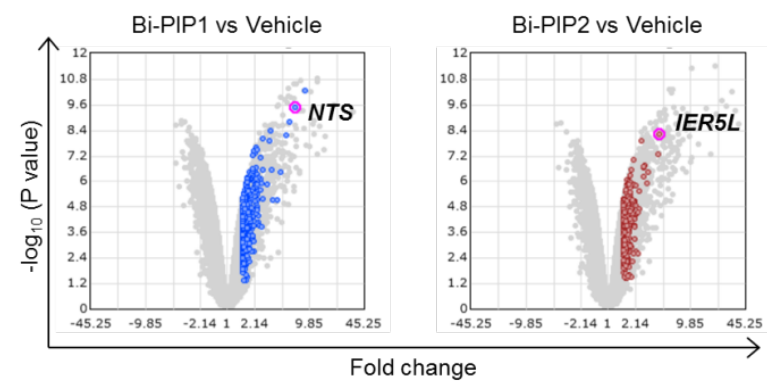

C

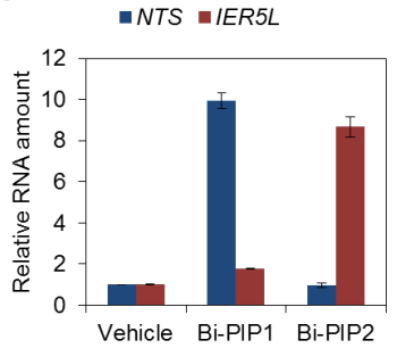

d
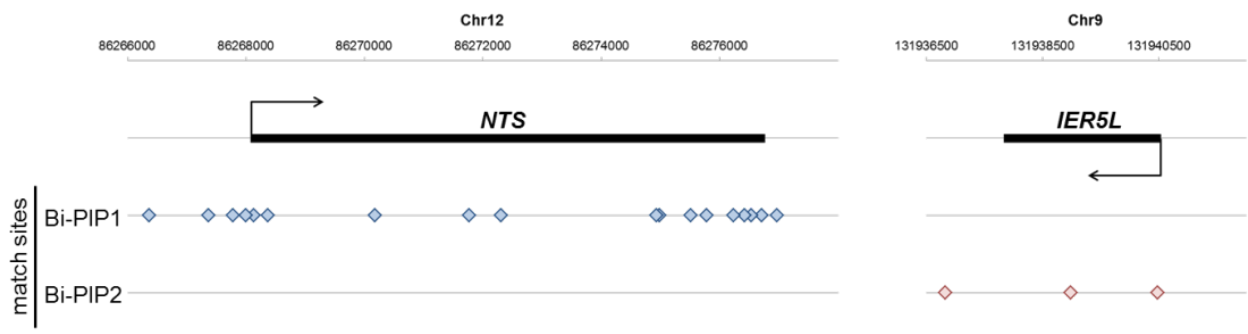

Figure 4. Bi-PIPs epigenetically activate selective gene expression inside living cells. (a) Motif search analysis. After ChIP-seq for acetylated $\mathrm{H}_{3}$, differential peaks between Bi-PIP1 and Bi-PIP2-treated cells were detected by ODIN. Each differential peak region was searched for the match sites of Bi-PIP1 (5'-WWCWGWCW-3', left) and Bi-PIP2 (5'-WGCCGCCW-3', right). (b) Volcano plots of transcriptome comparison of Bi-PIP1 vs. vehicle (left) and Bi-PIP2 vs. vehicle (right). Uniquely upregulated transcripts by individual Bi-PIP (>1.5 fold change) are indicated as colored dots. (c) Expression of NTS and IER $5 L$ was confirmed by RT-qPCR. Error bars represent standard deviation of data from two culture wells. (d) Genomic loci of NTS (upper) and IER5L (lower). Match sites for Bi-PIP1 (5'-WWCWGWCW-3') and Bi-PIP2 (5'-WGCCGCCW-3') are indicated as blue and red diamonds, respectively.

$\left(5^{\prime}-\mathrm{WWCWGWCW}-3^{\prime}\right)$ and Bi-PIP2 (5'-WGCCGCCW-3') in each gene locus. Interestingly, the genomic sequence of the NTS locus contains the binding sites for Bi-PIP1 but not for Bi-PIP2 (Figure $4 \mathbf{d}$ ). Likewise, the IER 5 L locus possesses the binding sites for Bi-PIP2 but not for Bi-PIP1 (Figure $4 \mathrm{~d}$ ). Furthermore, the differential peak analysis of ChIP-seq data between each Bi-PIP and vehicle-treated cells revealed that the acetylation at $I_{E} R_{5} L$ locus increased upon the Bi-PIP2 treatment and was detected as significant peaks by ODIN (Supplementary Figure 12). On the other hand, ODIN did not detect substantial peaks in the NTS locus. However, there was a notable change in the shape of the peak in the data (Supplementary Figure 12). This observation possibly reflects the chromatin remodeling or nucleosome eviction after Bi-PIPı induced acetylation at the locus. These results suggest that NTS and IER5 $L$ are putative target genes of $\mathrm{Bi}-\mathrm{PIP} 1$ and $\mathrm{Bi}-\mathrm{PIP} 2$, respectively.

\section{DISCUSSION}

Acetylated histones function by neutralizing the positive charge of lysine to loosen the chromatin structure, and by being recognized by BD-containing proteins. BDcontaining proteins are diverse nuclear proteins including HATs, histone methyltransferases, and ATP-dependent chromatin remodeling complexes. ${ }^{43}$ Recruiting these proteins at acetyl lysine sites thus contributes to gene activation by remodeling chromatin, ${ }^{44}$ introducing methylation at lysine 4 in histone $\mathrm{H}_{3}\left(\mathrm{H}_{3} \mathrm{~K}_{4} \mathrm{me}\right)$, or introducing de novo acetylation near the site. In this study, we sought to take advantage of the BD-mediated natural propagation system of histone acetylation for the control of targeted acetylation. With the reliable and robust molecular design, we successfully developed 'Bi-PIP,' which recruits $\mathrm{P}_{300 / C B P}$ to selective DNA sequences. Based on biochemical assays, Bi-PIP-mediated histone acetylation at target DNA sequences was demonstrated as a proof-of-concept of the molecular mechanism. Moreover, cellular experiments showed the sequence-selective histone acetylation and transcriptional activation in endogenous genome by $\mathrm{Bi}$ PIPs, indicating their applicability in living cells.

Recently Ansari's group reported a conjugate called SynTEF by coupling a PIP with another bromodomain inhibitor (+)-JQ1, selective to the bromodomain and extraterminal (BET) protein family. ${ }^{45}$ Syn-TEF1 targeting expanded GAA repeats in the frataxin $(F X N)$ gene successfully recruited BET protein $\mathrm{BRD}_{4}$ (bromodomain 4) to the gene locus in Friedreich's ataxia (FRDA) patient-derived cells, resulting in transcriptional elongation of FXN. However, Syn-TEF and Bi-PIP target different members of the BD protein family, and therefore is demonstrated to cause different outcomes i.e., while Syn-TEF causes BRD4dependent transcriptional elongation, Bi-PIP causes $\mathrm{P}_{3}$ oodependent histone acetylation. Thus, Bi-PIP and Syn-TEF can be used for different purposes and this make to expand the range of potential applications of the synthetic epigenetic regulators.

Despite the successful demonstration of the potential of Bi-PIP as biomimetic artificial epigenetic code, there is still 
a need to improve the sequence-selective capacity of the $\mathrm{Bi}$-PIPs inside the living cells. The reason is that the ChIPseq analysis of the Bi-PIP-treated cells implied that the acetylated loci by each Bi-PIP could contain not only its target sequences but also the other possible binding sites. Also, the microarray studies suggested that although some genes were selectively activated by each $\mathrm{Bi}$-PIP, others were activated by both Bi-PIPs. The modest selectivity of the current version of Bi-PIPs could be attributed to the non-exclusive distribution of the two target sequences on the genome-wide scale. Nevertheless, the next-generation Bi-PIPs with extended recognition capacity ${ }^{46}$ or those that are designed to have a cooperative binding effect ${ }^{47}$ could target the longer DNA sequences and overcome the nonselective outcome. Furthermore, a comprehensive evaluation of the binding affinity could facilitate the design of advanced Bi-PIPs with better specificity towards the loci of interest. ${ }^{48}$ Besides, the designer PIPs can also have an alternate application as the guiding factor for the $\mathrm{BD}$ inhibitor to target multiple short sequence motifs in the genome and epigenetically activate the desired gene network. 49

So far, we have developed a variety of PIP-based sequence-selective epigenetic regulators by employing small molecules that modulate epigenetic writers or erasers, including HDAC inhibitor (SAHA), ${ }^{8}$ HAT activator (CTB), ${ }^{16}$ and HAT inhibitor (C646). ${ }^{\circ}$ In this study, we expanded the utility of PIP-based sequence-selective epigenetic regulators to target epigenetic readers. Given that histone codes fundamentally function by being recognized by epigenetic readers, sequence-selective inhibitors of epigenetic readers such as Bi-PIP work as readable artificial histone codes while operating similarly as the natural ones. In the future, other inhibitors of epigenetic readers will also be employed to achieve diverse outcomes such as the introduction of other histone PTMs, removing histone PTMs, and chromatin remodeling.

\section{EXPERIMENTAL SECTION}

All experimental procedures are provided in the Supporting Information.

\section{ASSOCIATED CONTENT}

\section{Supporting Information}

The Supporting Information is available free of charge via the Internet at http://pubs.acs.org.

Synthesis and characterization of compounds

Experimental procedures

Sequence of DNA templates used for the nucleosomes

Supplementary Figures 1-12

Supplementary Tables 1-7

\section{AUTHOR INFORMATION}

\section{Corresponding Author}

* hs@kuchem.kyoto-u.ac.jp

\section{Funding Sources}

This study was supported by JSPS [16Ho6356 to H.S., 15Jo2111 to T.J., Kyoto University SPIRITS grant and 16K12896 to G.N.P.].

\section{Notes}

The authors declare no competing financial interest.

\section{ACKNOWLEDGMENT}

We thank Prof. Hideki Yorimitsu and Dr. Keisuke Nogi for supporting microwave-based chemical reactions conducted for the compound synthesis. We thank Prof. Yoshie Harada and Dr. YongWoon Han for providing plasmids for the expression of histones.

\section{REFERENCES}

(1) Grunstein, M. Histone Acetylation in Chromatin Structure and Transcription. Nature 1997, 389, 349-352.

(2) Dhalluin, C.; Carlson, J. E.; Zeng, L.; He, C.; Aggarwal, A. K.; Zhou, M. M. Structure and Ligand of a Histone Acetyltransferase Bromodomain. Nature 1999, 399 (6735), 491496.

(3) Yang, X. Lysine Acetylation and the Bromodomain: A New Partnership for Signaling. Bioessays 2004, 26, 1076-1087.

(4) Hilton, I. B.; D’Ippolito, A. M.; Vockley, C. M.; Thakore, P. I.; Crawford, G. E.; Reddy, T. E.; Gersbach, C. A. Epigenome Editing by a CRISPR-Cas9-Based Acetyltransferase Activates Genes from Promoters and Enhancers. Nat. Biotechnol. 2015, 33 (5), 510-517.

(5) Dervan, P. B.; Edelson, B. S. Recognition of the DNA Minor Groove by Pyrrole-Imidazole Polyamides. Curr. Opin. Struct. Biol. 2003, 13 (3), 284-299.

(6) Trauger, J. W.; Baird, E. E.; Dervan, P. B. Recognition of DNA by Designed Ligands at Subnanomolar Concentrations. Nature 1996, 382, 559-561.

(7) Turner, J. M.; Swalley, S. E.; Baird, E. E.; Dervan, P. B. Aliphatic/aromatic Amino Acid Pairings for Polyamide Recognition in the Minor Groove of DNA. J. Am. Chem. Soc. 1998, 120 (6), 6219-6226.

(8) Ohtsuki, A.; Kimura, M. T.; Minoshima, M.; Suzuki, T.; Ikeda, M.; Bando, T.; Nagase, H.; Shinohara, K. I.; Sugiyama, H. Synthesis and Properties of PI Polyamide-SAHA Conjugate. Tetrahedron Lett. 2009, 50 (52), 7288-7292.

(9) Pandian, G. N.; Nakano, Y.; Sato, S.; Morinaga, H.; Bando, T.; Nagase, H.; Sugiyama, H. A Synthetic Small Molecule for Rapid Induction of Multiple Pluripotency Genes in Mouse Embryonic Fibroblasts. Sci. Rep. 2012, 2, 544.

(10) Pandian, G. N.; Sato, S.; Anandhakumar, C.; Taniguchi, J.; Takashima, K.; Syed, J.; Han, L.; Saha, A.; Bando, T.; Nagase, H.; Sugiyama, H. Identification of a Small Molecule That Turns on the Pluripotency Gene Circuitry in Human Fibroblasts. ACS Chem. Biol. 2014, 9 (12), 2729-2736.

(11) Han, L.; Pandian, G. N.; Junetha, S.; Sato, S.; Anandhakumar, C.; Taniguchi, J.; Saha, A.; Bando, T.; Nagase, H.; Sugiyama, H. A Synthetic Small Molecule for Targeted Transcriptional Activation of Germ Cell Genes in a Human Somatic Cell. Angew. Chemie Int. Ed. 2013, 52 (50), 13410-13413.

(12) Syed, J.; Chandran, A.; Pandian, G. N.; Taniguchi, J.; Sato, S.; Hashiya, K.; Kashiwazaki, G.; Bando, T.; Sugiyama, H. A Synthetic Transcriptional Activator of Genes Associated with the Retina in Human Dermal Fibroblasts. ChemBioChem 2015, 16 (10), 1497-1501.

(13) Wei, Y.; Pandian, G. N.; Zou, T.; Taniguchi, J.; Sato, S.; Kashiwazaki, G.; Vaijayanthi, T.; Hidaka, T.; Bando, T.; Sugiyama, H. A Multi-Target Small Molecule for Targeted Transcriptional 
Activation of Therapeutically Significant Nervous System Genes. ChemistryOpen 2016, 5, 517-521.

(14) Pandian, G. N.; Taniguchi, J.; Junetha, S.; Sato, S.; Han, L.; Saha, A.; AnandhaKumar, C.; Bando, T.; Nagase, H.; Vaijayanthi, T.; Taylor, R. D.; Sugiyama, H. Distinct DNA-Based Epigenetic Switches Trigger Transcriptional Activation of Silent Genes in Human Dermal Fibroblasts. Sci. Rep. 2014, 4, 3843.

(15) Mantelingu, K.; Kishore, a H.; Balasubramanyam, K.; Kumar, G. V. P.; Altaf, M.; Swamy, S. N.; Selvi, R.; Das, C.; Narayana, C.; Rangappa, K. S.; Kundu, T. K. Activation of pзoo Histone Acetyltransferase by Small Molecules Altering Enzyme Structure : Probed by Surface-Enhanced Raman Spectroscopy. J. Phys. Chem. B 2007, 111, 4527-4534.

(16) Han, L.; Pandian, G. N.; Chandran, A.; Sato, S.; Taniguchi, J.; Kashiwazaki, G.; Sawatani, Y.; Hashiya, K.; Bando, T.; Xu, Y.; Qian, X.; Sugiyama, H. A Synthetic DNA-Binding Domain Guides Distinct Chromatin-Modifying Small Molecules to Activate an Identical Gene Network. Angew. Chemie Int. Ed. 2015, 54 (30), 8700-8703.

(17) Devipriya, B.; Parameswari, a. R.; Rajalakshmi, G.; Palvannan, T.; Kumaradhas, P. Exploring the Binding Affinities of p3oo Enzyme Activators CTPB and CTB Using Docking Method. Indian J. Biochem. Biophys. 2010, 47 (6), 364-369.

(18) Delvecchio, M.; Gaucher, J.; Aguilar-Gurrieri, C.; Ortega, E.; Panne, D. Structure of the p3oo Catalytic Core and Implications for Chromatin Targeting and HAT Regulation. Nat. Struct. Mol. Biol. 2013, 20 (9), 1040-1046.

(19) Yang, X.-J.; Ogryzko, V. V.; Nishikawa, J.; Howard, B. H.; Nakatani, Y. A p300/CBP-Associated Factor That Competes with the Adenoviral Oncoprotein E1A. Nature 1996, 382, 319-324.

(20) Wassarman, D. A.; Sauer, F. TAFII250: A Transcription Toolbox. J. Cell Sci. 2001, 114, 2895-2902.

(21) Devaiah, B. N.; Case-Borden, C.; Gegonne, A.; Hsu, C. H.; Chen, Q.; Meerzaman, D.; Dey, A.; Ozato, K.; Singer, D. S. $\mathrm{BRD}_{4}$ Is a Histone Acetyltransferase That Evicts Nucleosomes from Chromatin. Nat. Struct. Mol. Biol. 2016, 23 (6), 540-548.

(22) Nguyen, U. T. T.; Bittova, L.; Müller, M. M.; Fierz, B.; David, Y.; Houck-Loomis, B.; Feng, V.; Dann, G. P.; Muir, T. W. Accelerated Chromatin Biochemistry Using DNA-Barcoded Nucleosome Libraries. Nat. Methods 2014, 11 (8), 834-840.

(23) Li, S.; Shogren-Knaak, M. A. The Gen5 Bromodomain of the SAGA Complex Facilitates Cooperative and Cross-Tail Acetylation of Nucleosomes. J. Biol. Chem. 2009, 284 (14), 94119417.

(24) Filippakopoulos, P.; Qi, J.; Picaud, S.; Shen, Y.; Smith, W. B.; Fedorov, O.; Morse, E. M.; Keates, T.; Hickman, T. T.; Felletar, I.; Philpott, M.; Munro, S.; McKeown, M. R.; Wang, Y.; Christie, A. L.; West, N.; Cameron, M. J.; Schwartz, B.; Heightman, T. D.; Thangue, N. L.; French, C. A.; Wiest, O.; Kung, A. L.; Knapp, S.; Bradner, J. E. Selective Inhibition of BET Bromodomains. Nature 2010, 468 (7327), 1067-1073.

(25) Hay, D. A.; Fedorov, O.; Martin, S.; Singleton, D. C.; Tallant, C.; Wells, C.; Picaud, S.; Philpott, M.; Monteiro, O. P.; Rogers, C. M.; Conway, S. J.; Rooney, T. P. C.; Tumber, A.; Yapp, C.; Filippakopoulos, P.; Bunnage, M. E.; Müller, S.; Knapp, S.; Schofield, C. J.; Bradner, J. E. Discovery and Optimization of Small-Molecule Ligands for the CBP/p3oo Bromodomains. J. Am. Chem. Soc. 2014, 136 (26), 9308-9319.

(26) Fedorov, O.; Castex, J.; Tallant, C.; Owen, D. R.; Martin, S.; Aldeghi, M.; Monteiro, O.; Filippakopoulos, P.; Picaud, S.; Trzupek, J. D.; Gerstenberger, B. S.; Bountra, C.; Willmann, D.; Wells, C.; Philpott, M.; Rogers, C.; Biggin, P. C.; Brennan, P. E.; Bunnage, M. E.; Schüle, R.; Günther, T.; Knapp, S.; Müller, S. Selective Targeting of the BRG/PB1 Bromodomains Impairs Embryonic and Trophoblast Stem Cell Maintenance. Sci. Adv. 2015, 1 (10), e1500723.
(27) Theodoulou, N. H.; Bamborough, P.; Bannister, A. J.; Becher, I.; Bit, R. A.; Che, K. H.; Chung, C. W.; Dittmann, A.; Drewes, G.; Drewry, D. H.; Gordon, L.; Grandi, P.; Leveridge, M.; Lindon, M.; Michon, A. M.; Molnar, J.; Robson, S. C.; Tomkinson, N. C. O.; Kouzarides, T.; Prinjha, R. K.; Humphreys, P. G. Discovery of I-BRD9, a Selective Cell Active Chemical Probe for Bromodomain Containing Protein 9 Inhibition. J. Med. Chem. 2016, 59 (4), 1425-1439.

(28) Filippakopoulos, P.; Knapp, S. Targeting Bromodomains: Epigenetic Readers of Lysine Acetylation. Nat. Rev. Drug Discov. 2014, 13 (5), 337-356.

(29) Janknecht, R.; Hunter, T. A Growing Coactivator Network. Nature 1996, 383 (6595), 22-23.

(30) Hammitzsch, A.; Tallant, C.; Fedorov, O.; O’Mahony, A.; Brennan, P. E.; Hay, D. A.; Martinez, F. O.; Al-Mossawi, M. H.; de Wit, J.; Vecellio, M.; Wells, C.; Wordsworth, P.; Müller, S.; Knapp, S.; Bowness, P. $\mathrm{CBP}_{30}$, a Selective $\mathrm{CBP} / \mathrm{p} 300$ Bromodomain Inhibitor, Suppresses Human Thi7 Responses. Proc. Natl. Acad. Sci. 2015, 112 (34), 10768-10773.

(31) Muthurajan, U.; Mattiroli, F.; Bergeron, S.; Zhou, K.; Gu, Y.; Chakravarthy, S.; Dyer, P.; Irving, T.; Luger, K. In Vitro Chromatin Assembly: Strategies and Quality Control. Methods Enzymol. 2016, 573, 3-41.

(32) Lowary, P. T.; Widom, J. New DNA Sequence Rules for High Affinity Binding to Histone Octamer and Sequence-Directed Nucleosome Positioning. J. Mol. Biol. 1998, 276, 19-42.

(33) Luger, K.; Mäder, A. W.; Richmond, R. K.; Sargent, D. F.; Richmond, T. J. Crystal Structure of the Nucleosome Core Particle at $2.8 \AA$ Resolution. Nature 1997, 389, 251-26o.

(34) Thompson, P. R.; Wang, D.; Wang, L.; Fulco, M.; Pediconi, N.; Zhang, D.; An, W.; Ge, Q.; Roeder, R. G.; Wong, J.; Levrero, M.; Sartorelli, V.; Cotter, R. J.; Cole, P. A. Regulation of the p3oo HAT Domain via a Novel Activation Loop. Nat. Struct. Mol. Biol. 2004, 11 (4), 308-315.

(35) Karanam, B.; Jiang, L.; Wang, L.; Kelleher, N. L.; Cole, P. A. Kinetic and Mass Spectrometric Analysis of p3oo Histone Acetyltransferase Domain Autoacetylation. J. Biol. Chem. 2006, 281 (52), 40292-40301.

(36) Garcia, B. A.; Mollah, S.; Ueberheide, B. M.; Busby, S. A.; Muratore, T. L.; Shabanowitz, J.; Hunt, D. F. Chemical Derivatization of Histones for Facilitated Analysis by Mass Spectrometry. Nat. Protoc. 2007, 2 (4), 933-938.

(37) Lin, S.; Garcia, B. A. Examining Histone Posttranslational Modification Patterns by High-Resolution Mass Spectrometry. Methods Enzymol. 2012, 512, 3-28.

(38) Szerlong, H. J.; Prenni, J. E.; Nyborg, J. K.; Hansen, J. C. Activator-Dependent p3oo Acetylation of Chromatin in Vitro. J. Biol. Chem. 2010, 285 (42), 31954-31964.

(39) Best, T. P.; Edelson, B. S.; Nickols, N. G.; Dervan, P. B. Nuclear Localization of Pyrrole-Imidazole Polyamide-Fluorescein Conjugates in Cell Culture. Proc. Natl. Acad. Sci. U. S. A. 2003, 100 (21), 12063-12068.

(40) Nishijima, S.; Shinohara, K.; Bando, T.; Minoshima, M.; Kashiwazaki, G.; Sugiyama, H. Cell Permeability of Py-ImPolyamide-Fluorescein Conjugates: Influence of Molecular Size and Py/Im Content. Bioorg. Med. Chem. 2010, 18 (2), 978-983.

(41) Summerton, J. E. Endo-Porter: A Novel Reagent for Safe, Effective Delivery of Substances into Cells. Ann. N. Y. Acad. Sci. 2005, 1058, 62-75.

(42) Allhoff, M.; Sere, K.; Chauvistre, H.; Lin, Q.; Zenke, M.; Costa, I. G. Detecting Differential Peaks in ChIP-Seq Signals with ODIN. Bioinformatics 2014, 30 (24), 3467-3475.

(43) Muller, S.; Filippakopoulos, P.; Knapp, S. Bromodomains as Therapeutic Targets. Expert Rev. Mol. Med. 2o11, 13, e29.

(44) Awad, S.; Hassan, A. H. The Swiz/Snf2 Bromodomain Is Important for the Full Binding and Remodeling Activity of the 
SWI/SNF Complex on $\mathrm{H}_{3}$ - and $\mathrm{H}_{4}$-Acetylated Nucleosomes. Ann. N. Y. Acad. Sci. 2008, 1138 (1), 366-375.

(45) Erwin, G. S.; Grieshop, M. P.; Ali, A.; Qi, J.; Lawlor, M.; Kumar, D.; Ahmad, I.; Mcnally, A.; Teider, N.; Worringer, K.; Sivasankaran, R.; Syed, D. N.; Eguchi, A.; Jeffery, J.; Xu, M.; Park, P. M. C.; Mukhtar, H.; Srivastava, A. K.; Faruq, M.; Bradner, J. E.; Ansari, A. Z. Synthetic Transcription Elongation Factors License Transcription across Repressive Chromatin. Science (80-. ). 2017, 358 (6370), 1617-1622.

(46) Kawamoto, Y.; Sasaki, A.; Chandran, A.; Hashiya, K.; Ide, S.; Bando, T.; Maeshima, K.; Sugiyama, H. Targeting 24 Bp within Telomere Repeat Sequences with Tandem Tetramer Pyrrole-Imidazole Polyamide Probes. J. Am. Chem. Soc. 2016, 138, 14100-14107.

(47) Yu, Z.; Guo, C.; Wei, Y.; Hashiya, K.; Bando, T.; Sugiyama, H. Pip-HoGu: An Artificial Assembly with Cooperative
DNA Recognition Capable of Mimicking Transcription Factor Pairs. J. Am. Chem. Soc. 2018, 140 (7), 2426-2429.

(48) Erwin, G. S.; Bhimsaria, D.; Eguchi, A.; Ansari, A. Z. Mapping Polyamide-DNA Interactions in Human Cells Reveals a New Design Strategy for Effective Targeting of Genomic Sites. Angew. Chem. Int. Ed. Engl. 2014, 1-6.

(49) Soufi, A.; Garcia, M. F.; Jaroszewicz, A.; Osman, N.; Pellegrini, M.; Zaret, K. S. Pioneer Transcription Factors Target Partial DNA Motifs on Nucleosomes to Initiate Reprogramming. Cell 2015, 161 (3), 555-568.

(50) Yu, Z.; Taniguchi, J.; Wei, Y.; Pandian, G. N.; Hashiya, K.; Bando, T.; Sugiyama, H. Antiproliferative and Apoptotic Activities of Sequence-Specific Histone Acetyltransferase Inhibitors. Eur. J. Med. Chem. 2017, 138, 320-327. 
Bi-PIP:

Artificial Epigenetic Code of Acetylation

Acetyl lysine

mimetic

00000

D

DNA binder

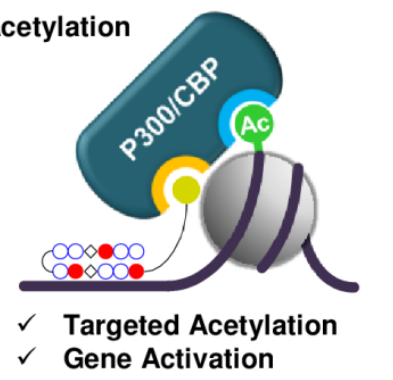

